\title{
Looking and listening: A comparison of intertrial repetition effects in visual and auditory search tasks
}

\author{
Michael D. Klein ${ }^{1}$ - Jennifer A. Stolz ${ }^{1}$
}

Published online: 6 May 2015

(C) The Psychonomic Society, Inc. 2015

\begin{abstract}
Previous research shows that performance on popout search tasks is facilitated when the target and distractors repeat across trials compared to when they switch. This phenomenon has been shown for many different types of visual stimuli. We tested whether the effect would extend beyond visual stimuli to the auditory modality. Using a temporal search task that has previously been shown to elicit priming of pop-out with visual stimuli (Yashar \& Lamy, Psychological Science, 21(2), 243-251, 2010), we showed that priming of pop-out does occur with auditory stimuli and has characteristics similar to those of an analogous visual task. These results suggest that either the same or similar mechanisms might underlie priming of pop-out in both modalities.
\end{abstract}

Keywords Visual search · Auditory search · Intertrial priming

Given the enormous amount of information continuously reaching us from the external world, we must efficiently select and process the most important of this information in order to survive and thrive. One approach to achieving this efficiency may be reflected in the finding that attention seems to be drawn towards features that have recently been the target of attention instead of new or recently ignored features (Kristjánsson \& Campana, 2010; Lamy, Antebi, Aviani, \& Carmel, 2008; Maljkovic \& Nakayama, 1994, 1996). In the context of popout search tasks (i.e., search tasks in which the target feature subjectively seems to "pop out" from the distractors, resulting

Michael D. Klein

mdklein@uwaterloo.ca

1 Department of Psychology, University of Waterloo, 200 University Ave. W., Waterloo, ON N2L 3G1, Canada in a search time that is largely uninfluenced by the number of distractors), this effect is called priming of pop-out (PoP) and manifests as shorter reaction times (RTs) when the target feature repeats across trials than when it switches.

The mechanisms underlying PoP seem to be quite general, as PoP has been observed for targets defined by many different kinds of features, including color (Maljkovic \& Nakayama, 1994), position (Geyer, Müller, \& Krummenacher, 2007; Maljkovic \& Nakayama, 1996), size (Huang, Holcombe, \& Pashler, 2004), shape (Thomson \& Milliken, 2011), feature dimension (Found \& Müller, 1996), relative shape (Becker, 2013), relative color (Becker, Valuch, \& Ansorge, 2014), and facial emotion (Lamy, Amunts, \& Bar-Haim, 2008), among others. One boundary condition may be that the localization of a target item amongst distractor items is necessary for the effect to emerge. For example, PoP might not occur when arrays of items are viewed passively (Kristjánsson, Saevarsson, \& Driver, 2013), when the location of the upcoming target is revealed by a spatial cue (Goolsby \& Suzuki, 2001), or when the target is presented in a constant location with no distractors (Wolfe, Butcher, Lee, \& Hyle, 2003).

Yashar and Lamy (2010) aimed to refine this boundary condition by testing whether spatial localization was necessary for PoP to occur, or whether the same effects would be expressed during a temporal search at a single spatial location. They used a rapid serial visual presentation (RSVP) task in which a sequence of 12 numbers appeared on screen one at a time. Eleven of the numbers were distractors that were presented in one color, and one was a differently colored target item. Participants indicated whether the target number was odd or even. The task involved temporal search because the target could be presented anywhere from the fifth to the ninth location on a given trial but did not involve spatial localization because all items appeared at the same spatial location. They found that PoP did occur in this temporal search task, with participants responding more 
quickly when the target and distractor colors repeated than when they switched. Additionally, they found that responses were faster when the temporal position of the target repeated than when it switched (temporal position priming), paralleling the common finding in spatial localization tasks of shorter RTs when the spatial location repeats. In a follow-up experiment, they showed that when temporal pop-out trials were interleaved with regular spatial pop-out trials, PoP occurred between the two types of trials, with temporal search leading to priming effects in the spatial task and spatial search leading to priming effects in the temporal task. This result suggests that PoP arises from mechanisms that are general to search in both the temporal and spatial domains.

We seek to further specify the generality of the mechanisms that underlie PoP. In particular, we ask whether these mechanisms generalize to the auditory modality. If so, we might expect PoP effects to be relatively independent of the modality of stimulus presentation, depending primarily on the type of task that is performed and not on the particular stimuli that are used. This would certainly be in line with the finding that PoP can be evoked using many different types of stimuli. If the mechanisms do not generalize, we might not expect PoP to occur at all in the auditory modality, or we might expect it to have different properties.

In the present study, we used the RSVP procedure described by Yashar and Lamy (2010) to compare temporal PoP between the visual (RSVP task) and auditory (rapid serial auditory presentation; RSAP) modalities. After finding a PoP effect in the RSAP task, we also used Yashar and Lamy's (2010) method of examining the relative contributions of the costs and benefits of target activation and distractor inhibition to get a clearer picture of any differences between the two modalities. That is, activation of the target item from the previous trial would benefit performance on the current trial if that item were to repeat as target (activation benefit) but would benefit cost performance if the item were to become the distractor (activation cost). Similarly, inhibition of the distractor item from the previous trial would benefit performance on the current trial if that item were to repeat as distractor (inhibition benefit) but would benefit cost performance if the item were to become the target (inhibition cost). Note that by the use of the words activation and inhibition we do not intend to make any assumptions about the actual mechanisms behind the effects (e.g., that there is actually something being activated or inhibited) but are simply using previously established terminology.

\section{Experiment 1}

\section{Method}

Participants Eighteen University of Waterloo undergraduate students (10 male, 8 female, $M_{\text {age }}=21.1, S D_{\text {age }}=1.5$ ) participated in the experiment for course credit. All reported normal or corrected-to-normal visual acuity and normal color vision, as well as normal hearing ability.

Design Within each task there was a within-subjects manipulation of whether the target on the current trial was the same as the target from the previous trial (repeated target condition), was the same as the distractor from the previous trial (switched distractor), or was not present during the previous trial (new target). Similarly, there was a within-subjects manipulation of whether the distractor on the current trial was the same as the distractor from the previous trial (repeated distractor), was the same as the target from the previous trial (switched target), or was not present during the previous trial (new distractor). All combinations of the above occurred during the experiment except for repeated target/switched distractor and repeated distractor/switched target, as those conditions would require the target and distractor to be the same as each other. Activation and inhibition costs and benefits were measured by comparing "switched" and "repeated" trial performance to "new" trial performance.

The position of the target within the rapid serial presentation sequence could also change by zero positions (i.e., a position repetition), one position, two positions, three positions, or four positions from one trial to the next. Repetition of target position was independent from target and distractor repetition.

Apparatus Displays were generated by an Intel Core i7 computer connected to a 24-in. LCD monitor with $640 \times 480$ resolution and a $60 \mathrm{~Hz}$ refresh rate. Audio was played through Logitech overear headphones. Responses were collected via keypress on the computer's keyboard. Participants viewed the display approximately $50 \mathrm{~cm}$ from the monitor and set the audio volume to a comfortable level.

Procedure The experiment consisted of a 30 minute auditory task and a 30 minute visual task, completed within the same session. Half of the participants completed the auditory task first, whereas the other half completed the visual task first. Each task consisted of 10 practice trials, followed by eight blocks of 40 experimental trials.

Visual Task The visual task closely followed the procedure of the RSVP task reported by Yashar and Lamy (2010). An RSVP stream was presented at the center of the screen and consisted of 12 successively presented combinations of two black letters (each $\left.1.1^{\circ} \times 1.1^{\circ}\right)$ in Courier font on a gray background, with a $100 \mathrm{~ms}$ presentation time for each item and a $50 \mathrm{~ms}$ interstimulus interval (ISI) between items. Each combination of letters spelled out one of four vowel sounds: "EE," "AY," "UH," or "OO." One of these four combinations was randomly chosen as the target for a given trial and was randomly presented one time at a position between the fifth and 
ninth (inclusive) of the sequence. Another combination was randomly chosen as the distractor for that trial and was presented at all of the other 11 positions. Each item within the sequence was presented slightly to the left or slightly to the right of center (shifted $1.1^{\circ}$ left or right for half of the participants and $2.2^{\circ}$ for the other half). The shift direction was randomly chosen for each item except for the second item in the stream, which was always on the opposite side from the first item, and the third item in the stream, which was always opposite from the second item. A vertical bar above and below the stream marked the center of the screen. A blank gray screen followed each RSVP sequence for $1500 \mathrm{~ms}$ until the beginning of the next sequence. A visualization of the procedure is shown in Fig. 1A. Participants were instructed to report whether the target was presented to the left or right of center by pressing the " $\mathrm{z}$ " key with their left index finger for a left presentation and by pressing the " $\mathrm{m}$ " key with their right index finger for a right presentation.

Auditory task The auditory task was the same as the visual task, except the stimuli were presented auditorily. Recordings of the four vowel sounds spoken at a medium rate by an American male voice were obtained using an open-source text-to-speech application on iSpeech.org, and the first $300 \mathrm{~ms}$ of each recording was sped up 3times to a duration of $100 \mathrm{~ms}$, without altering its pitch, using Audacity 2.0.3, an open-source sound editing software. Each item within the sequence was presented in either the left or the right ear, and participants were again instructed to report the side to which the target item was presented. A visualization of the auditory procedure is shown in Fig. 1C.

\section{Results}

All data from participants with less than $80 \%$ accuracy on either the visual ( 0 participants) or auditory ( 2 participants) task were removed from analysis. Trials with incorrect responses and trials immediately following incorrect responses (14.5\% of trials) as well as trials with RTs below $250 \mathrm{~ms}$ $(0.5 \%)$ or more than 3 standard deviations above the mean for a given subject and condition (1.7\%) were discarded from the RT analysis. The measured refresh rate of the computer monitor was $59.95 \mathrm{~Hz}$ for all participants. Mauchly's test of sphericity and Greenhouse-Geisser corrections are reported for all tests violating the assumption of sphericity.

Visual PoP Mean RTs and error rates for repeated, new, and switched targets and distractors in the visual modality are shown in Fig. 2. A paired-samples $t$ test between repeated and switched condition RTs revealed a significant PoP effect, $t(15)=4.06, S E=5.72, p=.001$, although there was no significant difference in error rates, $t<1$.

As in Yashar and Lamy (2010), activation and inhibition benefits were examined in a $2 \times 2$ analysis of variance (ANOVA) with Target Repetition (repeated vs. new) and Distractor Repetition (repeated vs. new) as factors. Four of the seven intertrial contingencies were included: repeated target/repeated distractor, repeated target/new
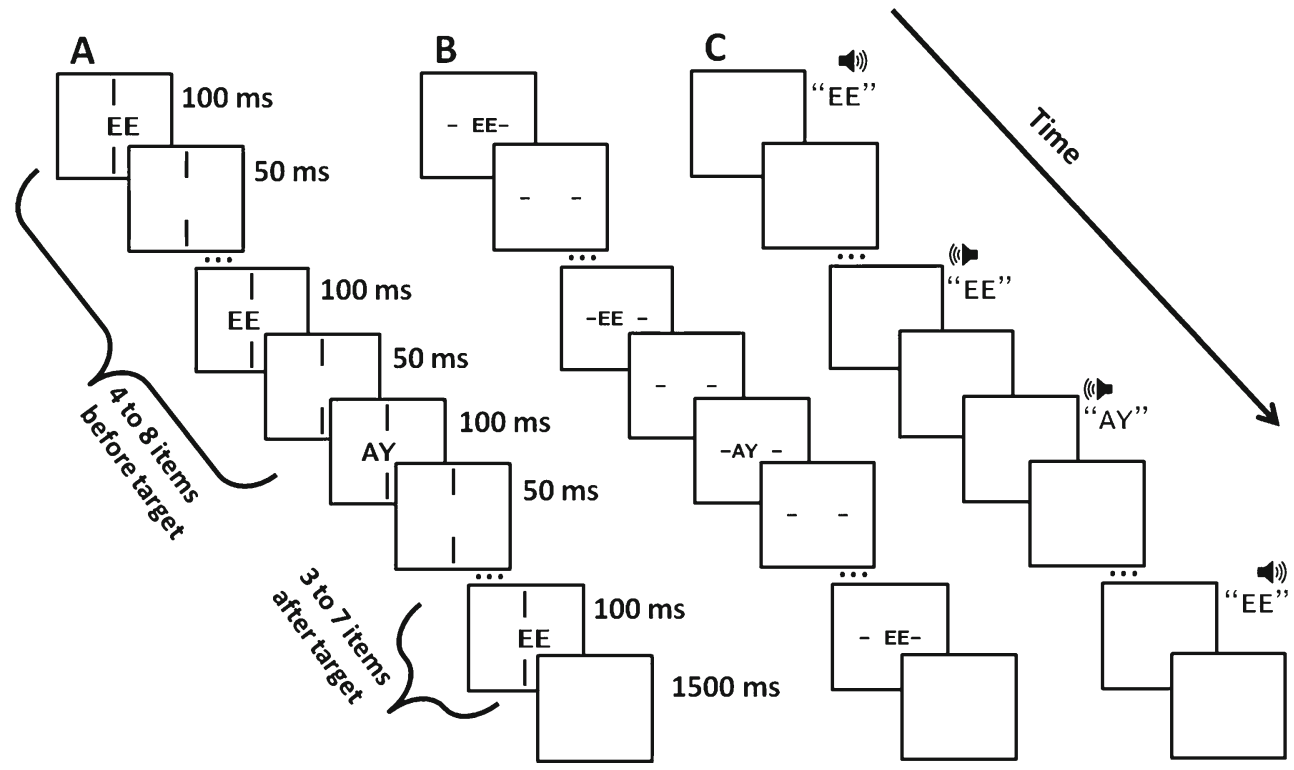

Fig. 1 An illustration of the procedure for Experiments 1 and 2. Twelve items were presented on each trial, with the target (oddball) item being presented between the fifth and ninth positions (inclusive). Each item was presented for $100 \mathrm{~ms}$, with a $50 \mathrm{~ms}$ interstimulus interval. A blank screen was presented for 1,500 ms after each trial. A. Items in the Experiment 1 visual task were spelled-out vowel sounds presented to the left or right of two vertical lines. B. Items in the Experiment 2 visual task were spelledout vowel sounds presented to the left or right of a space between two horizontal lines. C. Items in the Experiment 1 and 2 auditory tasks were spoken vowel sounds presented through headphones in either the left or right ear 


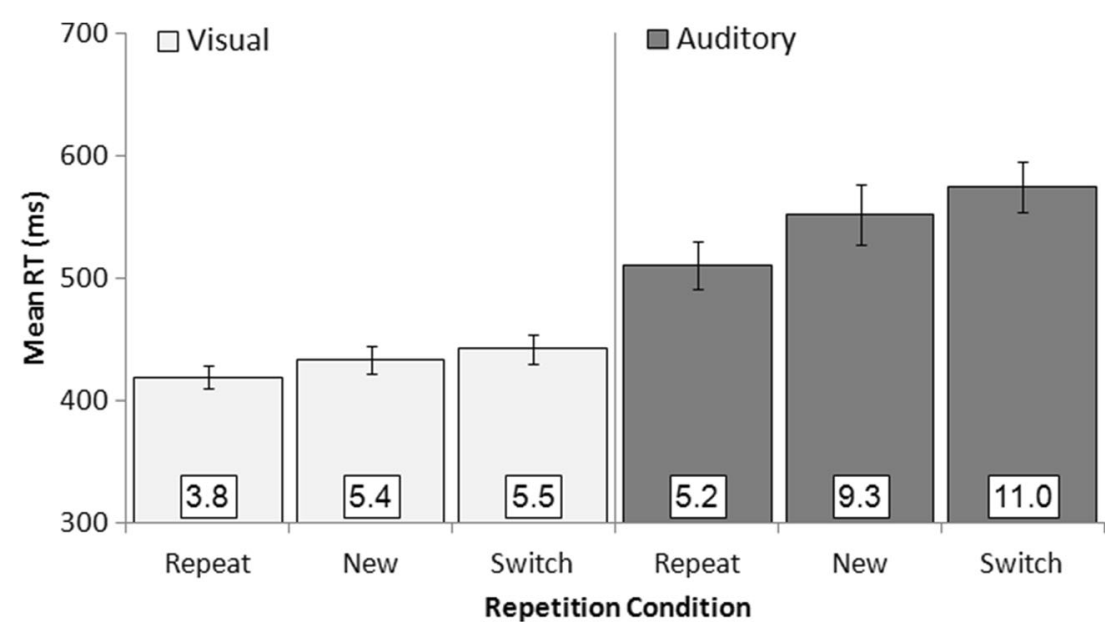

Fig. 2 Results from Experiment 1. Reaction time for trials on which the target and distractor repeated from the last trial (Repeat), trials on which the target and distractor were both items that were not present on the previous trial (New), and trials on which the target and distractor switched identities from the previous trial (Switch). Error bars represent 1 standard error above and below the mean. The number inside each bar is the error rate for that condition

Magnitude ( 0 positions vs. 1 position vs. 2 positions vs. 3 positions vs. 4 positions) as a factor. There was a significant effect of Position Change Magnitude, $F(1.91$, $28.71)=11.26, M S E=412.98, p<.001$, Mauchly's: $\chi^{2}(9)=30.1, p<.001$, Greenhouse-Geisser: $\varepsilon=0.48$. As shown in Fig. 4, RTs generally increased as the magnitude of position change increased. Based on a post hoc comparison using the Tukey HSD test, any RT mean differences greater than $20.93 \mathrm{~ms}$ were deemed significant at the .05 level $(r=5, d f=28.7)$. Using this criterion, RTs after a change of four positions were significantly longer than RTs after changes of zero, one, or two positions. No other differences were significant. A similar analysis using error rate data did not reveal a significant effect of position change magnitude, $F(4,60)=1.44, M S E=5.04$, $p=.233$.

Auditory PoP Mean RTs and error rates for repeated, new, and switched target and distractor in the auditory modality are shown in Fig. 2. A paired-samples $t$ test between repeated and switched conditions revealed a significant $\mathrm{PoP}$ effect in the RT data, $t(15)=4.21, S E=15.19, p=.001$, and a marginal error rate effect, $t(15)=2.01, S E=2.89, p=.063$, with slower reaction times and more errors for switched versus repeated targets and distractors.

Auditory activation and inhibition benefits were examined in the same fashion as the visual activation and inhibition benefits, with an ANOVA, including Target Repetition (repeated vs. new) and Distractor Repetition (repeated vs. new) as factors. The Target Switch $\times$ Distractor Switch interaction was not significant, $F<1$. As in the visual task, there was a significant main effect of repeated-versus-new distractor (inhibition benefit), $F(1,15)=5.56, M S E=1,053.00, p<.001$, with participants responding more quickly when the distractor 
Table 1 Mean and standard error for each intertrial contingency in Experiments 1 and 2

\begin{tabular}{|c|c|c|c|c|c|}
\hline \multirow[b]{2}{*}{ Condition } & & \multicolumn{2}{|l|}{ Experiment 1} & \multicolumn{2}{|l|}{ Experiment 2} \\
\hline & & Visual & Auditory & Visual & Auditory \\
\hline Target & Distractor & Mean $(S E)$ & Mean $(S E)$ & Mean $(S E)$ & Mean $(S E)$ \\
\hline New & New & $433.9(11.0)$ & $552.3(24.7)$ & $494.6(11.6)$ & $627.8(35.2)$ \\
\hline New & Repeated & $425.8(8.9)$ & $527.3(23.9)$ & $489.7(10.6)$ & $584.1(34.6)$ \\
\hline New & Switched & $435.5(9.9)$ & $538.2(27.3)$ & $493.2(10.9)$ & $617.9(31.9)$ \\
\hline Repeated & New & $430.4(9.2)$ & $557.7(28.0)$ & $492.6(11.9)$ & $610.6(32.9)$ \\
\hline Repeated & Repeated & $419.3(9.3)$ & $510.8(19.4)$ & $491.0(11.8)$ & $591.0(41.8)$ \\
\hline Switched & New & $435.5(10.7)$ & $568.6(29.1)$ & $504.3(12.2)$ & $659.1(37.8)$ \\
\hline Switched & Switched & $442.5(12.1)$ & $574.8(20.4)$ & $509.4(13.6)$ & $649.7(36.1)$ \\
\hline
\end{tabular}

identity repeated compared to when it was new. However, there was no effect of repeated-versus-new target (activation benefit), $F<1$.

Activation and inhibition costs were examined in a separate ANOVA, including Target Switch (switched vs. new) and Distractor Switch (switched vs. new) as factors. The Target Switch $\times$ Distractor Switch interaction was again not significant, $F<1$. There was a significant main effect of switchedversus-new target (inhibition cost), $F(1,15)=8.03, M S E=1$, $390.42, p=.013$, with participants responding more slowly when the target was switched rather than new. There was no effect of switched-versus-new distractor (activation cost), $F<$
1. The magnitude of each component of the auditory PoP effect is shown in Fig. 3.

The same analyses were carried out using the error rate data, which paralleled the RT data. There were no interactions, both $F_{\mathrm{S}}<1$, and no effect of repeated-versus-new target or switched-versus-new distractor, both $F_{\mathrm{s}}<1$. However, error rates were significantly lower for repeated than for new distractors, $F(1,15)=21.15, M S E=15.65, p$ $<.001$, and were significantly higher for switched than for new targets, $F(1,15)=9.19, M S E=16.39, p=.008$.

The change in temporal position of the target from the previous to the current trial was computed, and an ANOVA

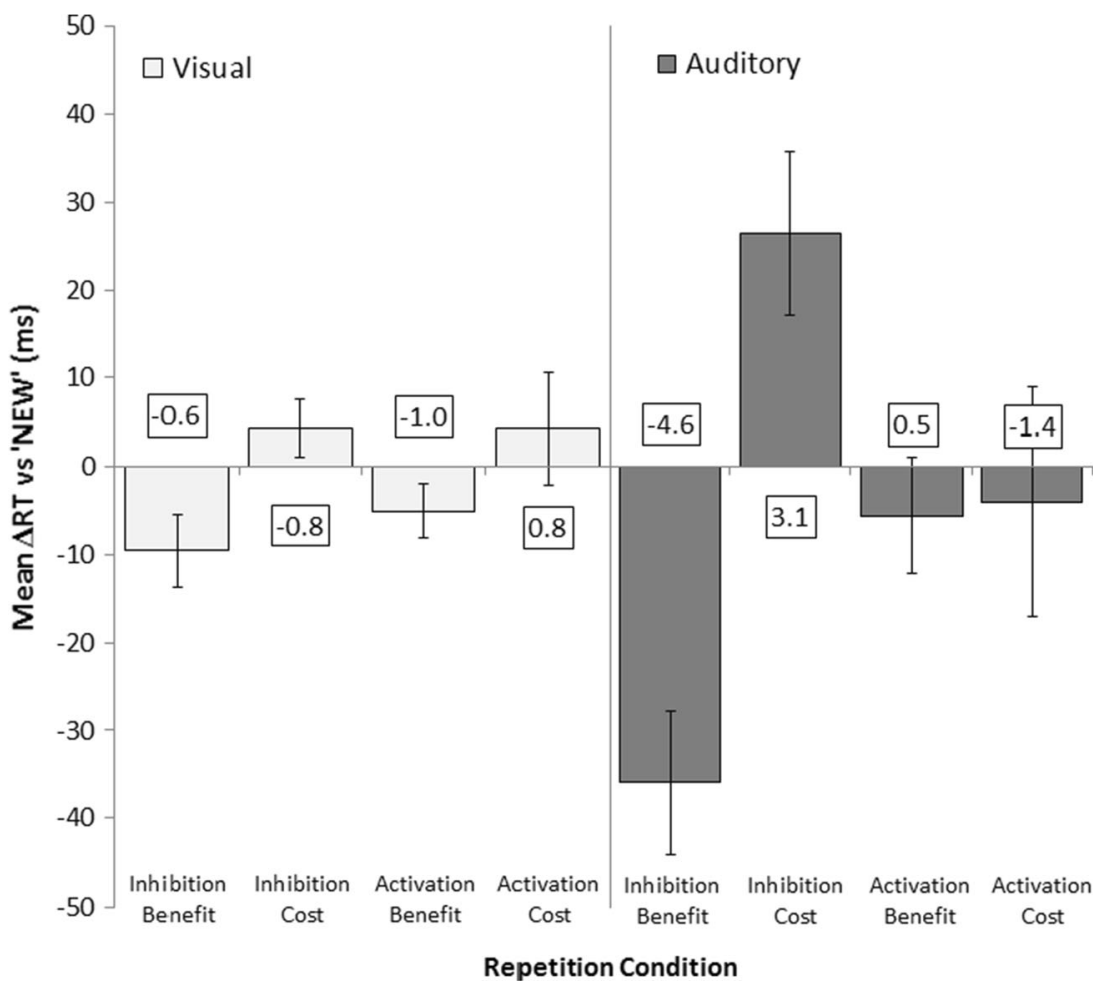

Fig. 3 Results from Experiment 1. Magnitude of the inhibition benefit (repeated minus new distractor RT), inhibition cost (switched minus new target RT) activation benefit (repeated minus new target RT), and activation cost (switched minus new distractor RT). Error bars represent
1 standard error above and below the mean. The number above or below each bar shows the error rate data (e.g., the number above inhibition benefit is the repeated distractor error rate minus the new distractor error rate) 


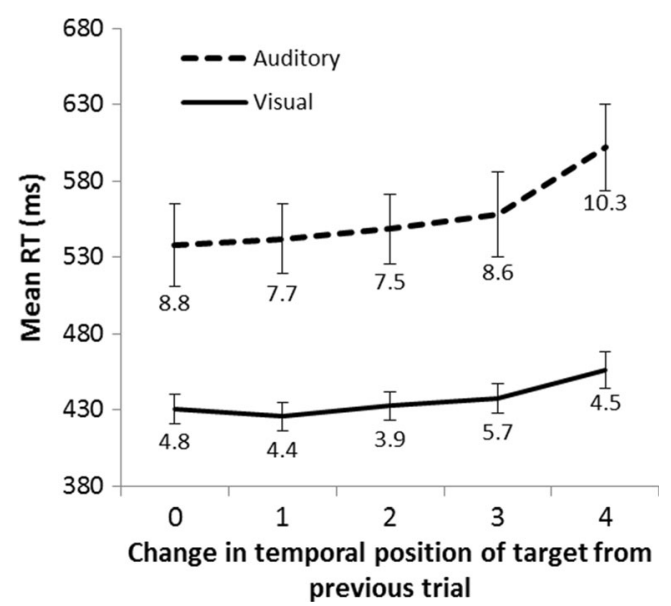

Fig. 4 Results from Experiment 1. Reaction time for trials on which the target position changed by zero through four positions from the previous trial. Error bars represent 1 standard error above and below the mean. The number below each point is the error rate for that condition

was performed using this Position Change Magnitude ( 0 positions vs. 1 position vs. 2 positions vs. 3 positions vs. 4 positions) as a factor. As in the visual task, there was a significant effect of Position Change Magnitude, $F(2.27,34.06)=$ 11.25, $M S E=1,673.33, p<.001$, Mauchly's: $\chi^{2}(9)=25.0, p=$ .003 , Greenhouse-Geisser: $\varepsilon=0.57$. As shown in Fig. 4 , the RTs for the auditory task also generally increased as the magnitude of position change increased. Based on a post hoc comparison using the Tukey HSD test, any RT mean differences greater than $41.64 \mathrm{~ms}$ were deemed significant at the .05 level $(r=5, d f=34.06)$. Using this criterion, RTs after a change of four positions were significantly longer than RTs after any other change in position. No other differences were significant. A similar analysis using error rate data did not reveal a significant effect of position change magnitude, $F<1$.

Visual versus auditory In order to compare visual with auditory PoP, RT data for each participant were divided by that participant's average RT for a new-target/newdistractor trial. An ANOVA was performed on these data using Modality (visual vs. auditory) and Repetition Condition (switched target and distractor vs. repeated target and distractor) as factors. There was no significant effect of Modality, $F<1$, but there was an effect of Repetition Condition, $F(1,15)=39.92, M S E=31.15, p<.001$, with faster responses for the repeated condition than for the switched condition. The Modality $\times$ Repetition Condition interaction was marginally significant, $F(1,15)=3.98$, $M S E=51.01, p=.065$, due to a larger difference between switch and repeat trials in the auditory modality (switch $=$ $105.5 \%$ of new-trial RT, repeat $=93.1 \%$ ) than in the visual modality $($ switch $=102.1 \%$, repeat $=96.9 \%$ ). Next, an ANOVA was performed using Modality (visual vs. auditory) and Component (activation benefit vs. activation cost vs. inhibition benefit vs. inhibition cost) as factors. There was no effect of Modality, $F<1$, but there was an effect of Component, $F(3,45)=14.19, M S E$ $=20.32, p<.001$, as well as a Modality $\times$ Component interaction, $F(3,45)=4.11, M S E=22.27, p=.012$. The interaction was characterized by an inhibition benefit that was significantly larger in the auditory modality, $t(15)=$ 2.53, $S E=1.57, p=.023$, as well as a larger inhibition cost in the auditory modality, $t(15)=2.41, S E=1.75, p=$ .029 , whereas the activation benefit, $t(15)=0.11, S E=$ $1.22, p=.916$, and the activation cost, $t(15)=0.32, S E=$ $2.91, p=.757$, did not differ between modalities.

To compare TPP between modalities, RT data for each participant were divided by that participant's average RT for a repeated-position trial. An ANOVA was performed on these data using Modality (visual vs. auditory) and Position Change Magnitude (1 position vs. 2 positions vs. 3 positions vs. 4 positions) as factors. There was a main effect of Modality, $F(1,15)=5.25, M S E=67.88$, $p=.037$, with a larger difference between repeated position and changed position RTs in the auditory modality than in the visual modality. There was also a main effect of Position Change Magnitude, $F(1.44,21.53)=20.81$, $M S E=25.17, p<.001$, Mauchly's: $\chi^{2}(5)=28.4, p<$ .001 , Greenhouse-Geisser: $\varepsilon=0.48$, with larger RTs for larger position changes. The Modality $\times$ Position Change Magnitude interaction was not significant, $F(1.71,25.61)$ $=2.37, M S E=16.40, p=.121$, Mauchly's: $\chi^{2}(5)=16.2$, $p=.007$, Greenhouse-Geisser: $\varepsilon=0.57$.

\section{Discussion}

The results of the visual component of Experiment 1 replicate Yashar and Lamy's (2010) finding of temporal search PoP and extend the finding from color priming to shape/letter priming. Similar to Yashar and Lamy (2010), this effect was driven only by distractor "inhibition" and not by target "activation." The results from the auditory component of Experiment 1 further extend the findings to the auditory modality, in which the PoP effect was also underlain only by distractor inhibition. There were differences between the modalities, however, as both item-repetition and positionrepetition effects were larger in the auditory than in the visual modality. Additionally, although a significant inhibition benefit was present in both modalities, an inhibition cost was present in only the auditory modality. Finally, although the auditory task led to significant priming effects in both the RT and error rate data, visual priming was apparent only in the RT data. It is not yet clear whether these differences reflect qualitative or quantitative differences in the processes that drive PoP in each modality. 


\section{Experiment 2}

In Experiment 1, the auditory task resulted in a larger overall PoP effect, including a greater inhibition benefit and inhibition cost than the visual task, as well as greater TPP. However, the auditory task was also more difficult, with longer reaction times and higher error rates. It is possible that this difference in difficulty, rather than any qualitative difference between auditory and visual PoP, caused the difference in the magnitude of the priming effect (see Maljkovic \& Nakayama, 1994). It is likely that the auditory task was more difficult in two ways: first, it was more difficult to identify which item was presented when it was spoken than when it was shown on the screen, and second, it was more difficult to determine which ear the target item had been presented to than it was to determine the side of the screen on which it had been displayed. For this reason, we increased the difficulty of these two components of the visual task in Experiment 2, whereas the auditory task was left the same.

\section{Method}

Participants Twenty-seven University of Waterloo undergraduate students (4 male, 23 female, $M_{\text {age }}=19.9, S D_{\text {age }}=$ 2.8) participated in the experiment for course credit. All reported normal or corrected-to-normal visual acuity and normal color vision, as well as normal hearing ability.

Design Experiment 2 used the same design as Experiment 1.

Apparatus Experiment 2 used the same apparatus as Experiment 1.

Procedure Experiment 2 used the same procedure as Experiment 1 except for some changes to the stimuli in the visual task. The font was changed from Courier to FixedSys, which is a smaller font (each letter subtended a visual angle of about $\left.0.9^{\circ} \times 0.6^{\circ}\right)$. The vertical bars that indicated the location of the center of the screen in Experiment 1 were also removed and replaced with dashes about $2.7^{\circ}$ to the left and to the right of center. Finally, each item in the sequence moved by only $0.6^{\circ}$ to the right or left of center, instead of by $1.1^{\circ}$ or $2.2^{\circ}$. A visualization of this procedure is shown in Fig. 1B.

\section{Results}

The measured refresh rate of the computer monitor was $59.95 \mathrm{~Hz}$ for all participants. Any block with accuracy more than 3 standard deviations below the mean for a given condition was removed, and any participant with more than two blocks removed from any one condition was completely removed from the analysis. This resulted in the removal of three participants plus $2.1 \%$ of the data. Trials with incorrect responses and trials immediately following incorrect responses ( $25 \%$ of trials) as well as trials with RTs below $250 \mathrm{~ms}(0.4 \%)$ or more than 3 standard deviations above the mean for a given subject and condition $(1.8 \%)$ were discarded from the RT analysis. The resulting mean RT was $613 \mathrm{~ms}$ in the auditory condition and $496 \mathrm{~ms}$ in the visual condition, while the overall error rate was $9.4 \%$ in the auditory condition and $15.8 \%$ in the visual condition.

Visual PoP Mean RTs and error rates for repeated, new, and switched targets and distractors in the visual modality are shown in Fig. 5. A paired-samples $t$ test between repeated and switched condition RTs revealed a significant PoP effect, $t(23)=2.30, S E=8.02, p=.031$, although there was no significant difference in error rates, $t<1$.

Activation and inhibition benefits were again examined in one analysis of variance (ANOVA), including Target Repetition (repeated vs. new) and Distractor Repetition (repeated vs. new) as factors, whereas activation and inhibition costs were examined in a separate ANOVA, including Target Switch (switched vs. new) and Distractor Switch (switched vs. new). As in Experiment 1, Target Switch $\times$ Distractor Switch interaction was not significant in either analysis, $F<1$. There was a significant main effect of switched-versus-new target (inhibition cost), $F(1,23)=7.36, M S E=548.64, p=.012$, with participants responding more slowly when the target identity switched compared to when it was new. No other effects were significant, all $F_{\mathrm{s}}<1$. The magnitude of each component of the visual PoP effect is shown in Fig. 6. The same analyses were carried out using the error rate data, however none of the main effects reached significance, all $p s>.15$.

To measure TPP, an ANOVA was performed with the Position Change Magnitude ( 0 positions vs. 1 position vs. 2 positions vs. 3 positions vs. 4 positions) as a factor. There was a significant effect of Position Change Magnitude, $F(1.39,31.97)=4.18, M S E=4,371.21, p=.037$, Mauchly's: $\chi^{2}(9)=84.1, p<.001$, Greenhouse-Geisser: $\varepsilon=0.35$. As shown in Fig. 7, RTs generally increased as the magnitude of position change increased. Based on a post hoc comparison using the Tukey HSD test, any RT mean differences greater than $32.62 \mathrm{~ms}$ were deemed significant at the .05 level $(r=5$, $d f=31.97)$. Using this criterion, RTs after a change of four positions were significantly longer than RTs after changes of zero, one, or two positions. No other differences were significant. A similar analysis using error rate data did not reveal a significant effect of position change magnitude, $F<1$.

Auditory PoP Mean RTs and error rates for repeated, new, and switched targets and distractors in the auditory modality are shown in Fig. 5. Paired-samples $t$ tests between repeated and switched conditions revealed a significant PoP effect in the RT data, $t(23)=3.24, S E=18.11, p=.004$, and in the error rate data, $t(23)=2.58, S E=2.24, p=.017$, with slower 


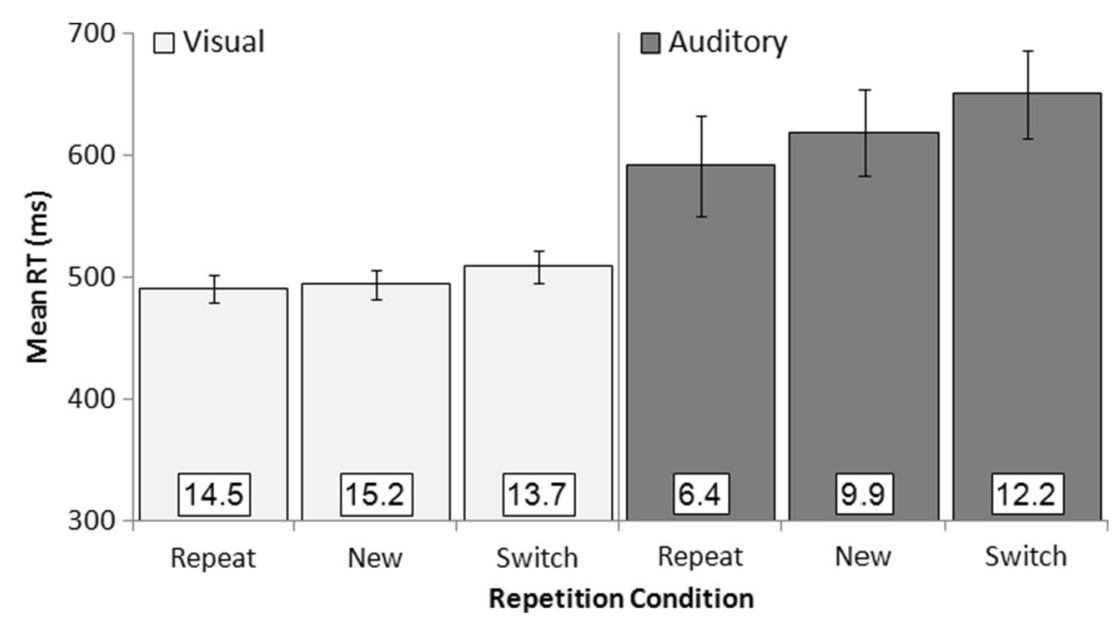

Fig. 5 Results from Experiment 2. Reaction time for trials on which the target and distractor repeated from the last trial (Repeat), trials on which the target and distractor were both items that were not present on the previous trial (New), and trials on which the target and distractor

reaction times and more errors for switched versus repeated targets and distractors.

Activation and inhibition benefits were examined in an ANOVA, including Target Repetition (repeated vs. new) and Distractor Repetition (repeated vs. new) as factors, and activation and inhibition costs were examined in a separate ANOVA, including Target Switch (switched vs. new) and switched identities from the previous trial (Switch). Error bars represent 1 standard error above and below the mean. The number inside each bar is the error rate for that condition

Distractor Switch (switched vs. new). The interaction between target and distractor effects was again nonsignificant in both analyses. As in Experiment 1, there was a significant effect of repeated-versus-new distractor (inhibition benefit), $F(1,23)=$ $6.65, M S E=2,566.19, p=.017$, with participants responding more quickly when the distractor identity repeated compared to when it was new, and a significant effect of switched-

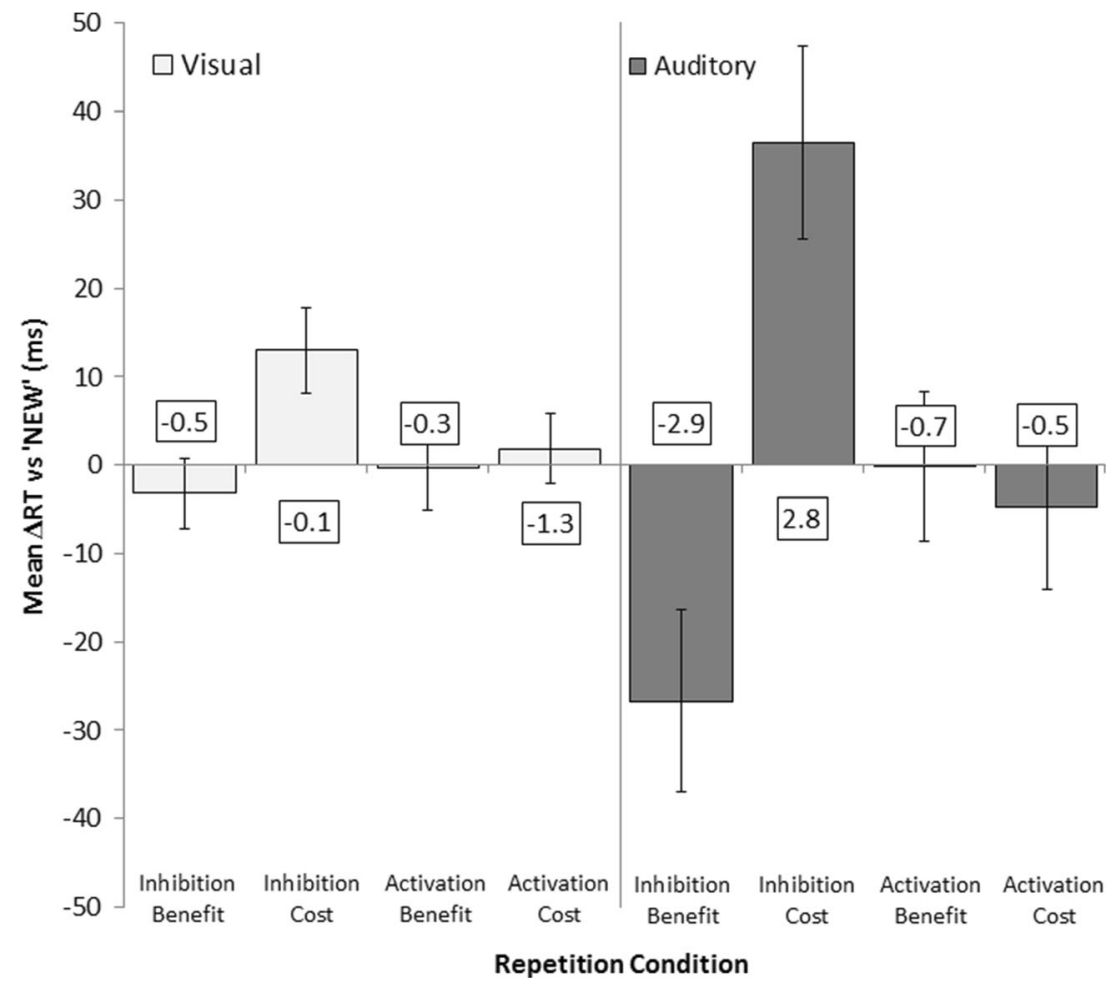

Fig. 6 Results from Experiment 2. Magnitude of the inhibition benefit (repeated minus new distractor RT), inhibition cost (switched minus new target RT) activation benefit (repeated minus new target RT), and activation cost (switched minus new distractor RT). Error bars represent
1 standard error above and below the mean. The number above or below each bar shows the error rate data (e.g., the number above inhibition benefit is the repeated distractor error rate minus the new distractor error rate) 


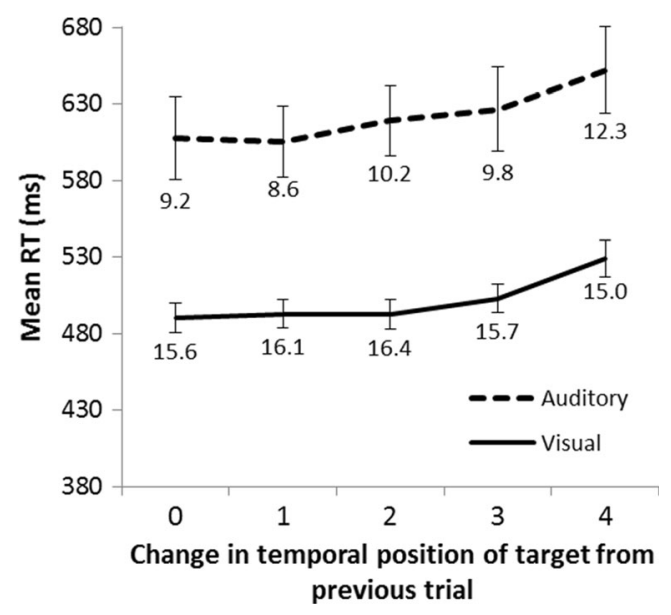

Fig. 7 Results from Experiment 2. Reaction time for trials on which the target position changed by zero through four positions from the previous trial. Error bars represent 1 standard error above and below the mean. The number below each point is the error rate for that condition

versus-new target (inhibition cost), $F(1,23)=11.15, M S E=2$, $868.17, p=.003$, with participants responding more slowly when the target was switched rather than new. There was no effect of switched-versus-new distractor (activation cost) or repeated-versus-new target (activation benefit), both $F_{\mathrm{S}}<1$. The magnitude of each component of the auditory PoP effect is shown in Fig. 6.

The same analyses were carried out using the error rate data. There were no interactions, both $F_{\mathrm{S}}<1$, and no effect of repeated-versus-new target or switched-versus-new distractor, both $F_{\mathrm{S}}<1$. However, error rates were marginally lower for repeated than for new distractors, $F(1,23)=3.98$, $M S E=50.71, p=.058$, and were marginally higher for switched than for new targets, $F(1,23)=3.81, M S E=$ $48.18, p=.063$.

The change in temporal position of the target from the previous to the current trial was computed, and an ANOVA was performed using the magnitude of this change ( 0 positions vs. 1 position vs. 2 positions vs. 3 positions vs. 4 positions) as a factor. There was a significant effect of Position Change Magnitude, $F(2.29,52.67)=4.52, M S E=3,269.09, p$ $=.012$, Mauchly's: $\chi^{2}(9)=40.3, p<.001$, GreenhouseGeisser: $\varepsilon=0.57$. Based on a post hoc comparison using the Tukey HSD test, any RT mean differences greater than $46.80 \mathrm{~ms}$ were deemed significant at the .05 level $(r=5$, $d f$ $=34.06)$. Using this criterion, none of the differences reached significance; however, as shown in Fig. 7, the RTs for the auditory task generally increased as the magnitude of position change increased. A similar analysis using error rate data yielded a marginal effect of Position Change Magnitude, $F(2.64,60.66)=2.78, M S E=26.30, p=.055$, Mauchly's: $\chi^{2}(9)=22.0, p=.009$, Greenhouse-Geisser: $\varepsilon=0.66$, with generally higher error rates for larger position changes. Based on a post hoc comparison using the Tukey HSD test, any RT mean differences greater than $4.17 \%$ were deemed significant at the .05 level $(r=5, d f=60.66)$, however none of the differences reached significance.

Visual versus auditory RT data for each participant were divided by that participant's average RT for a new-target/ new-distractor trial. An ANOVA was performed on these data using Modality (visual vs. auditory) and Repetition Condition (switched target and distractor vs. repeated target and distractor) as factors. There was no significant effect of Modality, $F<1$, but there was an effect of Repetition Condition, $F(1,23)=20.93, M S E=57.66, p<.001$, with faster responses for the repeated condition than for the switched condition. The Modality $\times$ Repetition Condition interaction was marginally significant, $F(1,23)=4.03, M S E=80.41, p=.056$, due to a larger difference between switch and repeat trials in the auditory modality (switch $=105.7 \%$ of new-trial RT, repeat $=$ $95.0 \%$ ) than in the visual modality (switch $=102.9 \%$, repeat $=99.5 \%$ ). Next, an ANOVA was performed using Modality (visual vs. auditory) and Component (activation benefit vs. activation cost vs. inhibition benefit vs. inhibition cost) as factors. There was no effect of Modality, $F<1$, but there was an effect of Component, $F(3,69)=11.09, M S E=$ $35.886, p<.001$, as well as a Modality $\times$ Component interaction, $F(3,69)=3.48, M S E=35.20, p=.020$. The interaction was characterized by an inhibition benefit that was significantly larger in the auditory modality, $t(23)=2.45, S E=1.77, p=$ .022 . The inhibition cost was numerically larger in the auditory modality but did not reach significance, $t(23)=1.61, S E=$ $2.15, p=.120$, whereas the activation benefit and the activation cost did not differ between modalities, both $t \mathrm{~s}<1$.

To compare temporal position PoP between modalities, RT data for each participant were divided by that participant's average RT for a repeated-position trial. An ANOVA was performed on this data using Modality (visual vs. auditory) and Position Change Magnitude (1 position vs. 2 positions vs. 3 positions vs. 4 positions) as factors. There was no effect of Modality, $F<1$; however, there was a main effect of Position Change Magnitude, $F(1.45,33.28)=10.10, M S E=0.01, p=$ .001 , Mauchly's: $\chi^{2}(5)=42.3, p<.001$, Greenhouse-Geisser: $\varepsilon=0.48$, with larger RTs for larger position changes. The Modality $\times$ Position Change Magnitude interaction was not significant, $F<1$.

Asymmetry of temporal position priming Yashar and Lamy (2013) studied temporal position priming more rigorously by analyzing the data for each temporal position separately, finding TPP for earlier but not for later current-trial target positions. They argued that TPP was reduced due to floor effects caused by the decrease in uncertainty of the target position as more positions passed with no target. If auditory TPP is caused by similar underlying mechanisms, we should expect the RSAP task to produce a similar pattern of results. Figure 8, which shows RT for each combination of current and 


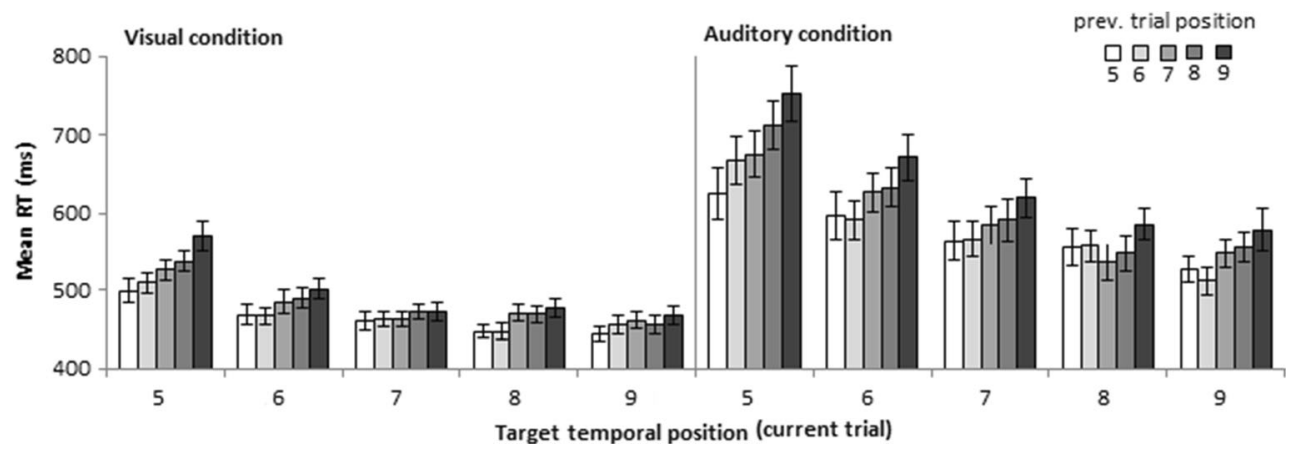

Fig. 8 Results from Experiments 1 and 2. Reaction time as a function of both current trial target position and previous trial target position. In both the visual and the auditory tasks there was strong temporal position priming for early but not for late current-trial target positions. Error bars represent 1 standard error above and below the mean. previous trial target position, suggests that TPP in both tasks did wane at later target positions. We combined the data from Experiments 1 and 2, and for both the visual and auditory tasks conducted an ANOVA with Current Trial Target Position (5 vs. 9) and Previous Trial Target Position (5 vs. 6 vs. 7 vs. 8 vs. 9) as factors. For both the visual and auditory tasks, there was a main effect of Current Position, visual: $F(1,39)=$ 107.69, $M S E=4,672.71, p<.001$, auditory: $F(1,39)=$ 899.90, $M S E=22,547.00, p<.001$, and a main effect of Previous Position, visual: $F(4,156)=10.11, M S E=2$, 502.23, $p<.001$, auditory: $F(4,156)=12.93, M S E=7$, 943.45, $p<.001$. The Current Position $\times$ Previous Position interaction was marginally signification for the visual task, $F(2.55,99.34)=2.65, M S E=5,144.02, p=.062$, Mauchly's: $\chi^{2}(9)=38.5, p<.001$, Greenhouse-Geisser: $\varepsilon=0.64$, and significant for the auditory task, $F(4,156)=2.75, M S E=6$, $357.74, p=.030$. The effect of Previous Trial Target Position remained significant when the target was in the ninth position on the current trial in the auditory modality, $F(2.95,115.00)=$ 5.87, MSE $=6,256.13, p=.001$, Mauchly's: $\chi^{2}(9)=23.86, p=$ .005 , Greenhouse-Geisser: $\varepsilon=0.74$, but not in the visual modality, $F(4,156)=1.72, M S E=1,779.26, p=.148$. This suggests that TPP in both modalities was driven by a tendency to respond more slowly when the target appeared later in the previous trial, and that this tendency was reduced, but not necessarily eliminated, when the target appeared later in the current trial.

\section{Discussion}

The results from the auditory task in Experiment 2 replicate our finding of a PoP effect using auditory stimuli, which is driven by both costs and benefits of distractor inhibition and exists in both the RT and error rate data. In the visual modality, the increase in difficulty did not have a large influence on RTs but did result in a much higher error rate. The auditory task still resulted in significantly greater overall PoP than the visual task, as well as a larger inhibition benefit; however, the magnitude of the inhibition cost was not significantly larger in the auditory modality, nor was the magnitude of TPP. Despite the high error rates on the visual task, there was still no evidence of PoP in the visual error rate data. Also of note is the fact that in the visual modality, an inhibition cost occurred but an inhibition benefit did not, whereas the reverse was true in Experiment 1.

\section{General Discussion}

The main goal of the experiments reported here was to assess whether the mechanisms underlying priming of pop-out generalize to the auditory modality. Across two experiments using the rapid serial presentation paradigm described by Yashar and Lamy (2010), we demonstrated PoP using both visually presented spelled-out vowel sounds and auditorily presented spoken vowel sounds as stimuli. In both modalities, PoP occurred for both item repetition and temporal position repetition, with participants responding more quickly when the target and distractor repeated than when they switched, and more quickly when the position of the target within the sequence changed by fewer positions from one trial to the next than when it changed by more positions.

These effects do not seem to be the result of auditory repetition priming (e.g., Bergerbest, Ghahremani, \& Gabrieli, 2004; Dyson, 2010), which is the finding that participants respond more quickly to attributes of a sound that was recently heard compared to attributes of a new sound. Auditory repetition priming would be expected to show up as an activation benefit (i.e., a difference between repeated vs. new target trials), which did not occur in our experiments. This is important because analogous repetition priming effects tend not to occur in visual PoP experiments, in which target localization, and not simply response to a stimulus, is necessary to elicit priming (Goolsby \& Suzuki, 2001; Kristjánsson et al., 2013; Wolfe et al., 2003). Therefore it is likely that our visual 
and auditory tasks measured the same thing - that is, priming resulting from the temporal discrimination of a target amongst distractors.

One persistent difference between the two modalities is that the effect of item repetition was larger in the auditory modality. This difference cannot be fully explained by differences in task difficulty between the two modalities because when the difficulty of the visual task was increased in Experiment 2, the difference persisted. However, despite this increased difficulty, participants still responded more quickly to the visual task than they did to the auditory task. It is possible that the smaller effect size was simply a consequence of the shorter RTs in the visual condition, although this seems unlikely for two reasons. First, the difference persisted even when the data were normalized by new-target/new-distractor RT; the percentage change compared to new-target/new-distractor trials was still larger in the auditory modality. Second, priming effects never showed up in the visual error rate data, even though the error rates in Experiment 2 were high enough to avoid floor effects. This suggests that PoP in the visual modality was simply not strong enough to influence error rate, which is generally less sensitive to manipulation than RT.

Another noteworthy difference between the modalities is the relative contributions of distractor inhibition costs and benefits. In both the auditory and visual modalities, PoP consisted of only distractor inhibition effects, with no target activation effects. However, in the auditory modality, both a cost and a benefit of inhibition occurred in each experiment, whereas in the visual modality only a benefit of inhibition occurred in Experiment 1, and only a cost of inhibition occurred in Experiment 2. It is not clear why such a small change to the visual task would result in a cost occurring instead of a benefit. One potential explanation for this finding is that because of the smaller magnitude of PoP in the visual modality, the individual components cannot be measured as reliably. That is, given the small difference in RT between "switched" and "repeated" distractor trials in the visual modality ( $\sim 15 \mathrm{~ms}$ ), the cost/benefit breakdown is much more sensitive to slight differences in the RT of "new" distractor trials, whereas in the auditory modality a small change in the RT of "new" distractor trials would have little influence due to the large difference in RT between "switched" and "repeated" distractor trials ( $\sim 60 \mathrm{~ms})$. Perhaps if the magnitude of PoP could be increased, a reliable cost and benefit of inhibition would both be observed (see Yashar \& Lamy, 2010, who found both a cost and benefit in a similar task using color as the target-defining feature).

It cannot be concluded with certainty whether the above differences in PoP between the auditory and visual modalities reflect qualitative or quantitative differences in the processes that underlie PoP in each modality. However, it is clear that
PoP does occur during temporal search for both visual and auditory stimuli, and that it has very similar characteristics in each modality.

Given these similarities, future research should continue to analyze these characteristics by looking for similarities and differences across a wider range of tasks and by determining the underlying causes of any differences between the modalities. Another question for future research is whether PoP plays a similar functional role in both modalities. Maljkovic and Nakayama (2000) proposed that PoP plays an important role in the guidance of focal visual attention and saccadic eye movements by facilitating return to recent targets of attention. This function does not befit the RSAP and RSVP tasks for two reasons. First, these tasks do not involve eye movements, and second, given that we found evidence for inhibition of distractor items but not for activation of target items, the facilitation of return to recent targets of attention seems not to have been a factor in temporal PoP. Both auditory and visual temporal PoP instead seem to have been driven by mechanisms that suppress attention to repeated distracting items, allowing focus to be placed on any different target item, whether repeated or new. However, this is a distinction between temporal and spatial PoP. Whether there is also a functional difference between visual and auditory PoP remains to be seen.

\section{References}

Becker, S. I. (2013). Simply shapely: Relative, not absolute shapes are primed in pop-out search. Attention, Perception, \& Psychophysics, 75(5), 845-861.

Becker, S. I., Valuch, C., \& Ansorge, U. (2014). Color priming in pop-out search depends on the relative color of the target. Frontiers in Psychology, 5, 289.

Bergerbest, D., Ghahremani, D. G., \& Gabrieli, J. D. (2004). Neural correlates of auditory repetition priming: Reduced fMRI activation in the auditory cortex. Journal of Cognitive Neuroscience, 16(6), 966-977.

Dyson, B. J. (2010). Trial after trial: General processing consequences as a function of repetition and change in multidimensional sound. The Quarterly Journal of Experimental Psychology, 63(9), 1770-1788.

Found, A., \& Müller, H. J. (1996). Searching for unknown feature targets on more than one dimension: Investigating a "dimension-weighting" account. Perception \& Psychophysics, 58(1), 88-101.

Geyer, T., Müller, H. J., \& Krummenacher, J. (2007). Cross-trial priming of element positions in visual pop-out search is dependent on stimulus arrangement. Journal of Experimental Psychology: Human Perception and Performance, 33(4), 788.

Goolsby, B. A., \& Suzuki, S. (2001). Understanding priming of colorsingleton search: Roles of attention at encoding and "retrieval". Perception \& Psychophysics, 63(6), 929-944.

Huang, L., Holcombe, A. O., \& Pashler, H. (2004). Repetition priming in visual search: Episodic retrieval, not feature priming. Memory \& Cognition, 32(1), 12-20. 
Kristjánsson, Á., \& Campana, G. (2010). Where perception meets memory: A review of repetition priming in visual search tasks. Attention, Perception, \& Psychophysics, 72(1), 5-18.

Kristjánsson, Á., Saevarsson, S., \& Driver, J. (2013). The boundary conditions of priming of visual search: From passive viewing through task-relevant working memory load. Psychonomic Bulletin \& Review, 20(3), 514-521.

Lamy, D., Amunts, L., \& Bar-Haim, Y. (2008a). Emotional priming of pop-out in visual search. Emotion, 8(2), 151.

Lamy, D., Antebi, C., Aviani, N., \& Carmel, T. (2008b). Priming of pop-out provides reliable measures of target activation and distractor inhibition in selective attention. Vision Research, 48(1), 30-41.

Maljkovic, V., \& Nakayama, K. (1994). Priming of pop-out: I. Role of features. Memory \& Cognition, 22(6), 657-672.

Maljkovic, V., \& Nakayama, K. (1996). Priming of pop-out: II. The role of position. Perception \& Psychophysics, 58(7), 977-991.
Maljkovic, V., \& Nakayama, K. (2000). Priming of pop-out: III. A shortterm implicit memory system beneficial for rapid target selection. Visual Cognition, 7(5), 571-595.

Thomson, D. R., \& Milliken, B. (2011). A switch in task affects priming of pop-out: Evidence for the role of episodes. Attention, Perception, \& Psychophysics, 73(2), 318-333.

Wolfe, J. M., Butcher, S. J., Lee, C., \& Hyle, M. (2003). Changing your mind: On the contributions of top-down and bottom-up guidance in visual search for feature singletons. Journal of Experimental Psychology: Human Perception and Performance, 29(2), 483.

Yashar, A., \& Lamy, D. (2010). Intertrial repetition facilitates selection in time common mechanisms underlie spatial and temporal search. Psychological Science, 21(2), 243-251.

Yashar, A., \& Lamy, D. (2013). Temporal position priming: Memory traces of recent experience bias the allocation of attention in time. Journal of Experimental Psychology: Human Perception and Performance, 39(5), 1443-1456. 